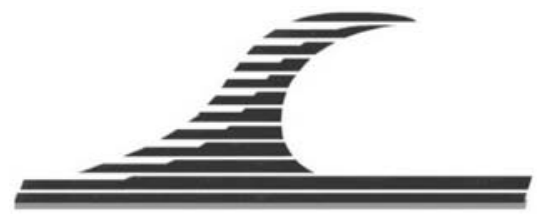

Revue Paralia, Volume 11 (2018) pp s01.1-s01.13

Mots-clés : Génie côtier, Hydraulique maritime, Travaux

maritimes, Environnement littoral, Digue, Franchissement.

(C) Editions Paralia CFL

\title{
Conception des extensions urbaines en mer. Spécificités et défis
}

\section{Pierre ARISTAGHES ${ }^{1}$}

1. Directeur Technique Travaux Maritimes de Bouygues TP, 1 avenue Freyssinet, 78280 - Guyancourt, France.

p.aristaghes@bouygues-construction.com

\section{Résumé :}

Le présent article évoque les spécificités de la conception de ce type d'ouvrage par rapport à des ouvrages portuaires classiques. Elles sont principalement liées à la recherche systématique d'ouvrages aussi proches que possible de la mer par beau temps, tout en en assurant confort et sécurité acceptables par tempête. Ceci entraîne d'une part à rechercher des solutions aussi peu encombrantes ou visibles que possible pour réduire les franchissements, et d'autre part à traiter les problèmes liés à des structures basses exposées à la houle, problèmes que l’on cherche en général à éviter.

\section{Design of urban extensions at sea. Specificities and challenges}

\begin{abstract}
:
The design of offshore urban extensions presents specificities compared to port structures design. In particular, the search for minimal overall height for architectural and urbanistic reasons leads to new solutions for overtopping management, which in turn lead to new types of structural problems.
\end{abstract}

\section{Key words:}

Coastal Engineering, Maritime hydraulics, Maritime works, Littoral environment, Breakwater, Overtopping. 


\section{Contexte général}

Les zones littorales sont de manière générale intensément peuplées, et la tendance de l'urbanisation du littoral au niveau mondial ne semble pas devoir s'inverser. Ceci est d'autant plus problématique dans le contexte de changement climatique en cours, du fait de la surélévation du niveau général des mers qui l'accompagne, mais aussi, même si le sujet est moins quantifiable, de la possible augmentation des événements extrêmes avec les risques d'érosion et de submersion associés.

Certaines villes sont pour des raisons diverses confrontées à la nécessité de s'étendre "sur la mer". Monaco en est un exemple remarquable, puisque le manque de place l'a conduit dans les années passées à augmenter sa superficie de $25 \%$ en gagnant $2 \mathrm{~km}^{2}$ sur la mer, et ceci par des fonds de plus de $30 \mathrm{~m}$, quand les extensions en mer similaires restaient dans des profondeurs en général beaucoup plus faibles, seuls des projets japonais ayant atteint les $20 \mathrm{~m}$ (BOUCHET, 2007). Le projet en cours de l'Anse du Portier (ARISTAGHES et al., 2018), qui augmentera de 6 ha la surface de la Principauté, atteint les profondeurs d'eau de l'ordre de $35 \mathrm{~m}$.

\section{Compromis entre proximité à la mer et protection en cas de tempête}

\subsection{Contexte général}

Les projets d'extension urbaines en mer ont en commun la recherche d'une cote d'arase aussi basse que possible pour des raisons diverses : proximité à la mer par beau temps, maintien de la vue sur la mer depuis des endroits emblématiques.

Dans le cas du Front de Mer de Beyrouth (ARISTAGHES \& CHALOIS, 2007), il n’était pas envisageable qu'il soit plus haut que le front de mer préexistant, ceci bien que l'extension en mer se fasse sur une zone plus exposée aux houles que le front de mer existant, situé au fond d'une baie naturellement protégée des plus fortes houles.

Par rapport au design d'un ouvrage portuaire, le concepteur doit trouver des compromis, consistant par exemple à définir des périodes de non-accès lorsque c'est possible, ou des solutions alternatives innovantes. La qualité du dialogue entre ingénieur et architecte est, plus encore que pour d'autres types d'ouvrages, essentielle pour que chacun comprenne les souhaits et contraintes de l'autre.

\subsection{Murs de crête optimisés ou maquillés}

La première solution consiste à concevoir des murs chasse-mer optimisés d'une manière ou d'une autre, ou à leur donner un autre usage qui facilite leur intégration dans le projet architectural.

Dans le cas où l'ouvrage est de type digue à talus, une première solution consiste à déporter le mur chasse-mer en arrière de la crête de talus, voire à ajouter en arrière du mur une "tranchée drainante", dans l’objectif d'atteindre des débits de franchissements acceptables tout en restant en dessous d'une altitude limite. 
Ceci est illustré par la marina qui terminait le front de mer de Beyrouth, et qui était censée être peu franchie pour des houles décennales de hauteur $\mathrm{H}_{\mathrm{mo}}=7.4 \mathrm{~m}$ et de période $\mathrm{T}_{\mathrm{p}}=15 \mathrm{~s}$ avec un niveau d'eau à $\mathrm{Z}_{\mathrm{w}}=+0.8 \mathrm{~m} \mathrm{CD}$, ceci sans interrompre la vue sur la mer d'un hôtel qui allait se retrouver en fond de marina. La figure 1 montre le concept retenu, sur la base d'une approche "balistique".

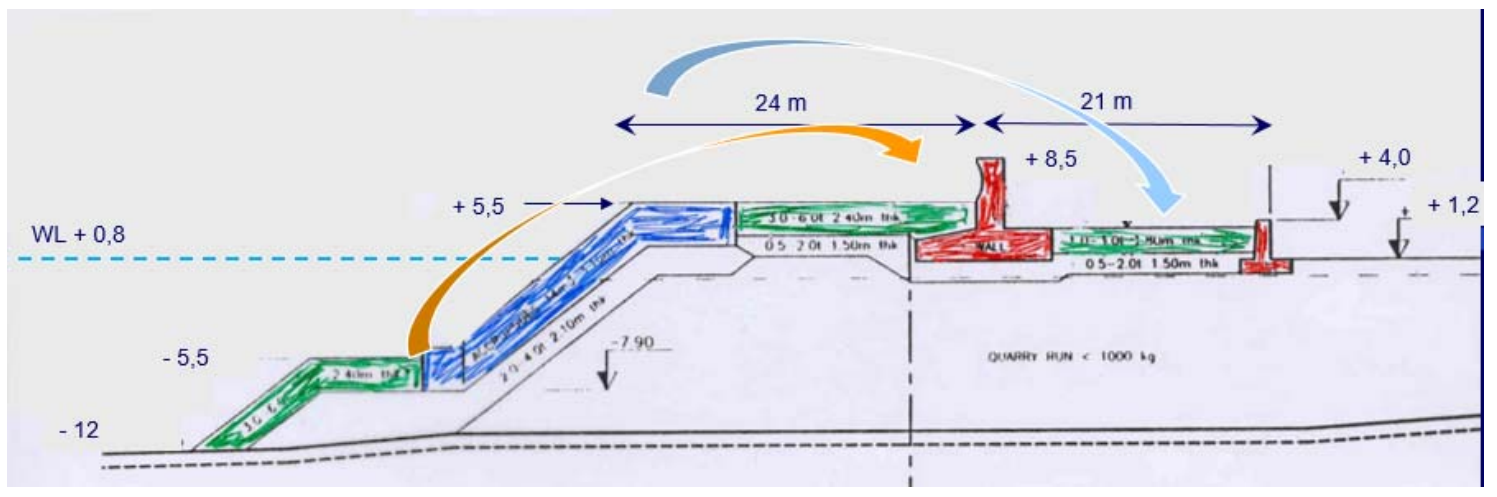

Figure 1. Coupe digue marina front de mer de Beyrouth.

Une autre solution consiste à concevoir le mur chasse-mer de manière à ce qu'on oublie sa fonction hydraulique. Un bon exemple en est donné par le front de mer de Beyrouth, où le parapet chasse-mer, au départ refusé par le maître d'ouvrage qui imposait une dalle directement ouverte à la mer, a finalement été accepté dès lors qu’il était dessiné comme un banc continu le long de la promenade. Ce concept, représenté en figure 2 a été imaginé par l'architecte Louis Sato, qui s’est lui-même inspiré d'un aménagement similaire en pied de l'opéra de Sydney.

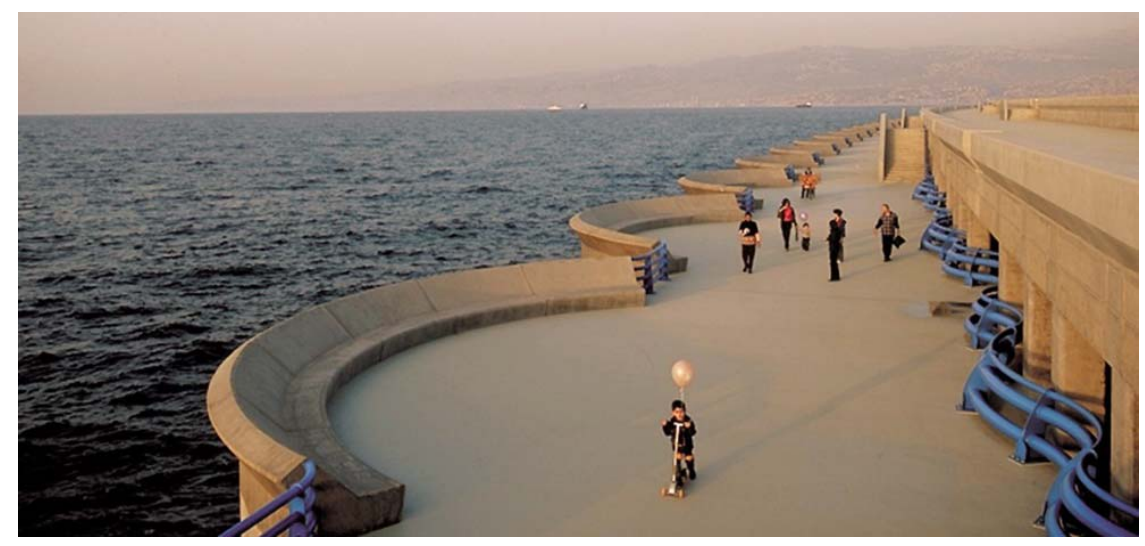

Figure 2. Promenade du front de mer de Beyrouth.

\subsection{Dispositifs absorbant les franchissements}

Une autre solution consiste à essayer d'absorber les vagues plutôt que de tenter de les arrêter (via un mur) ou de les laminer (via un glacis en enrochements). 
De ce point de vue, les structures en caissons se prêtent bien à l'inclusion de chambres d'absorption ou de dissipation des vagues. La chambre constitue un volume de stockage dans laquelle s'engouffre une part significative du volume d'eau franchissant en crête de vague, et qui la renvoie vers la mer par gravité entre deux crêtes successives.

Une variante aux chambres jarlan a été utilisée pour la protection du Palais des Congrès de Monaco. Il s’agit du concept breveté par Artelia des chambres anti-réflexion ARC, où la vidange de la chambre lors du creux s'accompagne d'un mouvement tourbillonnaire provoqué par la forme des cavités (figure 3).

Pour des dimensions adaptées aux longueurs d'onde des vagues à atténuer, ces concepts réduisent les coefficients de réflexion, les efforts hydrodynamiques et, ce qui nous intéresse plus directement, les franchissements. Cependant, la paroi, même perforée, tend à canaliser une partie de l'écoulement vers le haut, ce qui contribue aux franchissements.
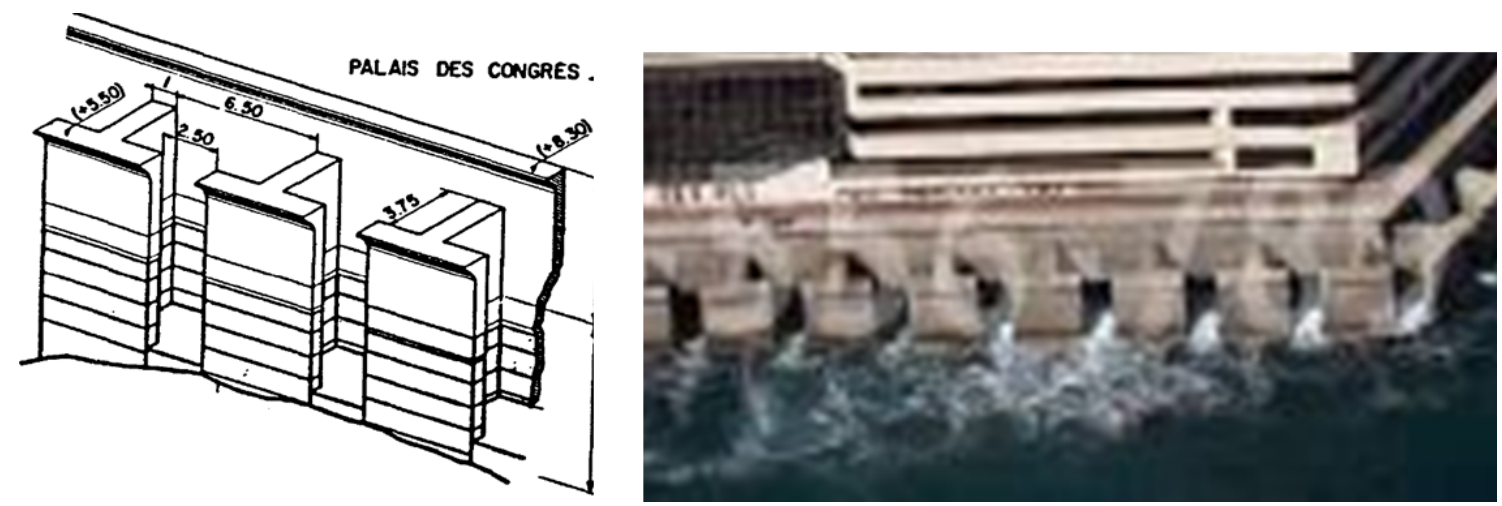

Figure 3. Caissons ARC et protection du Palais des Congrès de Monaco.

Pour réduire encore ceux-ci, une solution radicale serait de supprimer toute paroi, ce qui est impossible si l'on veut soutenir ou contenir une extension urbaine qui ne soit pas une plate-forme sur pieux ou flottante. Une réponse partielle à cet objectif est néanmoins apportée par la "trappe à vagues", à savoir un volume de stockage dans lequel la vague vient directement se jeter. Ce concept a été mis en oeuvre entre les deux promenades du front de mer de Beyrouth. Ceci est illustré par les caissons du front de mer de Beyrouth représentés au paragraphe précédent, et dont la vue éclatée de la figure 4 montre les différents éléments contribuant à la limitation des franchissements. 


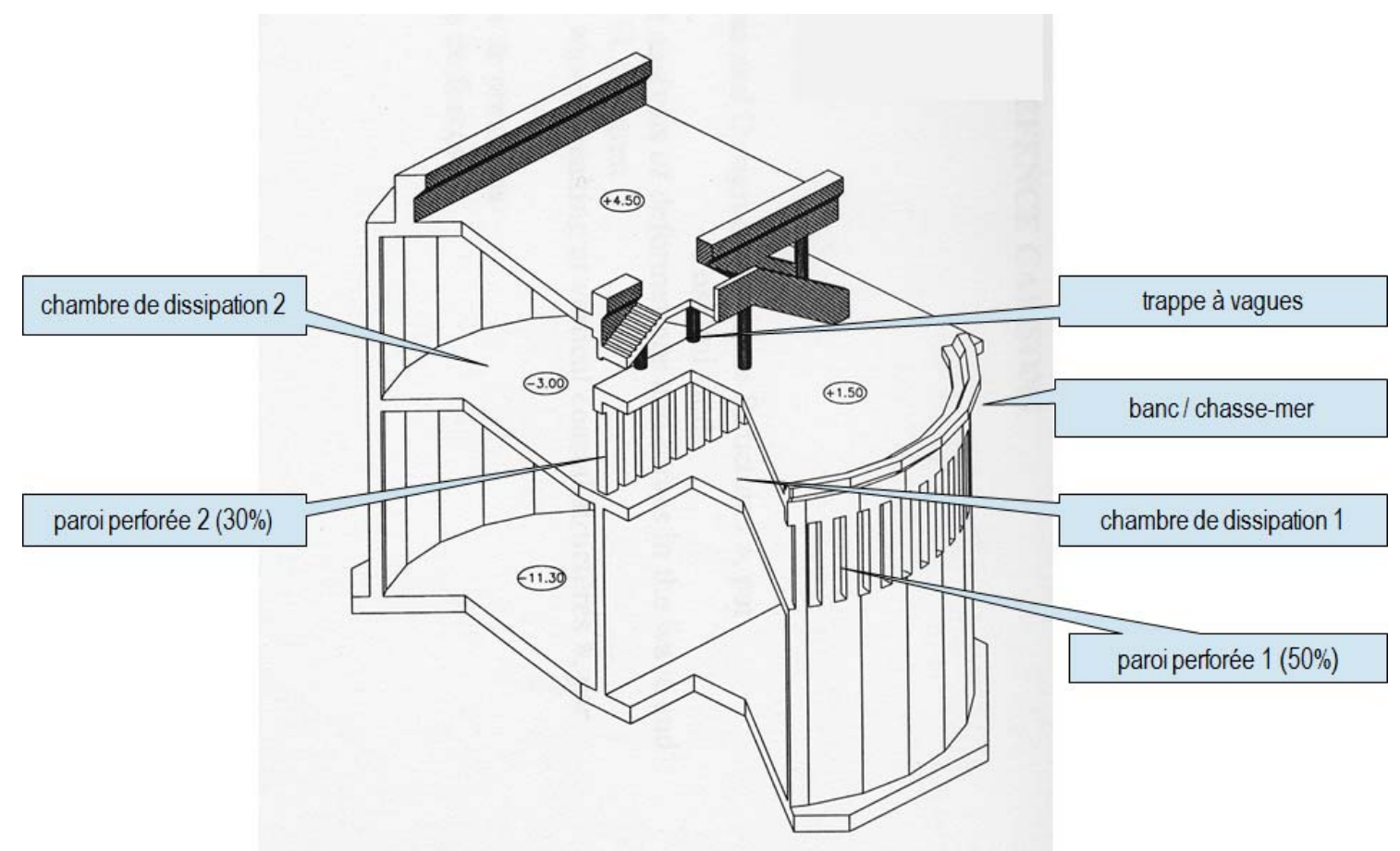

Figure 4. Vue éclatée du caisson multi-chambres du front de mer de Beyrouth.

Ceci est illustré par les caissons du front de mer de Beyrouth représentés au paragraphe précédent, et dont la vue éclatée suivante montre les différents éléments contribuant à la limitation des franchissements.

La trappe à vagues s'est révélée lors des essais en modèle réduit particulièrement efficace pour limiter les franchissements sur la promenade haute, du fait de l'absence de paroi verticale entre les niveaux de promenade. Elle nécessite par contre un garde-corps à la fois robuste et transparent aux vagues, ce qui a conduit, là encore en liaison étroite avec l'architecte, à concevoir une structure similaire aux "miséricordes" que l'on trouve dans les stalles de cathédrales (structure bleue de la photo précédente).

Alors que les chambres jarlan classiques sont en partie immergées, on peut également limiter les franchissements via des "chambres perchées", utilisées notamment dans le cas des digues de Tanger Méditerranée (figure 5). 


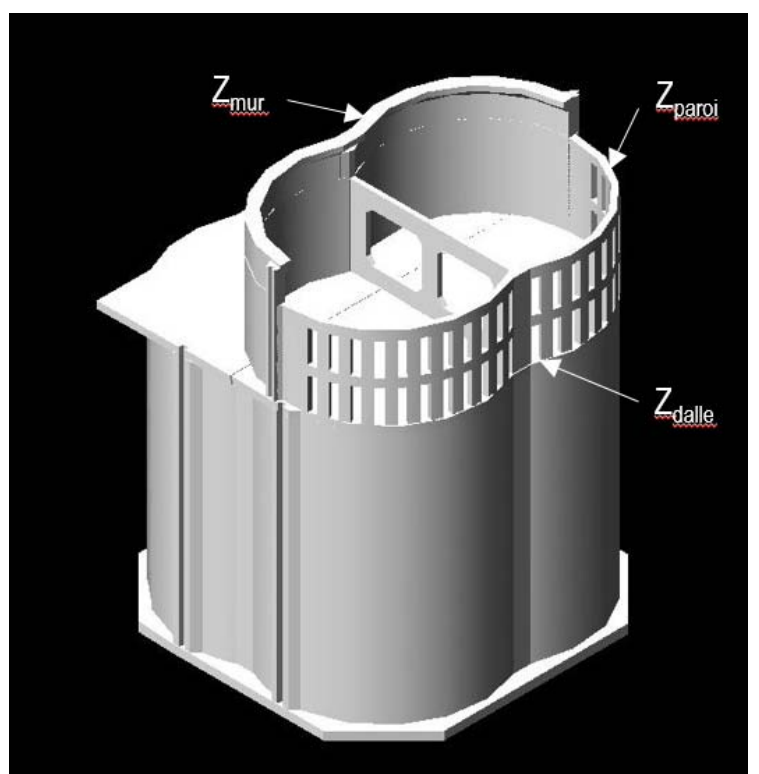

Figure 5. Caisson à chambre perchée

De nombreux essais en canal ont été effectués à l'occasion de ces projets (chez Océanide et à l'université du Havre), en faisant varier non seulement les houles et les niveaux d'eau mais aussi les trois niveaux apparaissant sur la figure précédente, et en testant également l'effet d'un déflecteur en tête de mur arrière.

Le graphe de la figure 6 montre, pour une partie de ces essais où la dalle avait été positionnée à $2.5 \mathrm{~m}$ au-dessus du niveau d'eau à pleine mer, la corrélation entre les débits mesurés et les débits calculés pour une digue verticale de niveau de crête égal à $\mathrm{Z}_{\mathrm{mur}}$ selon les formules de l'Eurotop dans sa version de 2016 (EUROTOP, 2016).

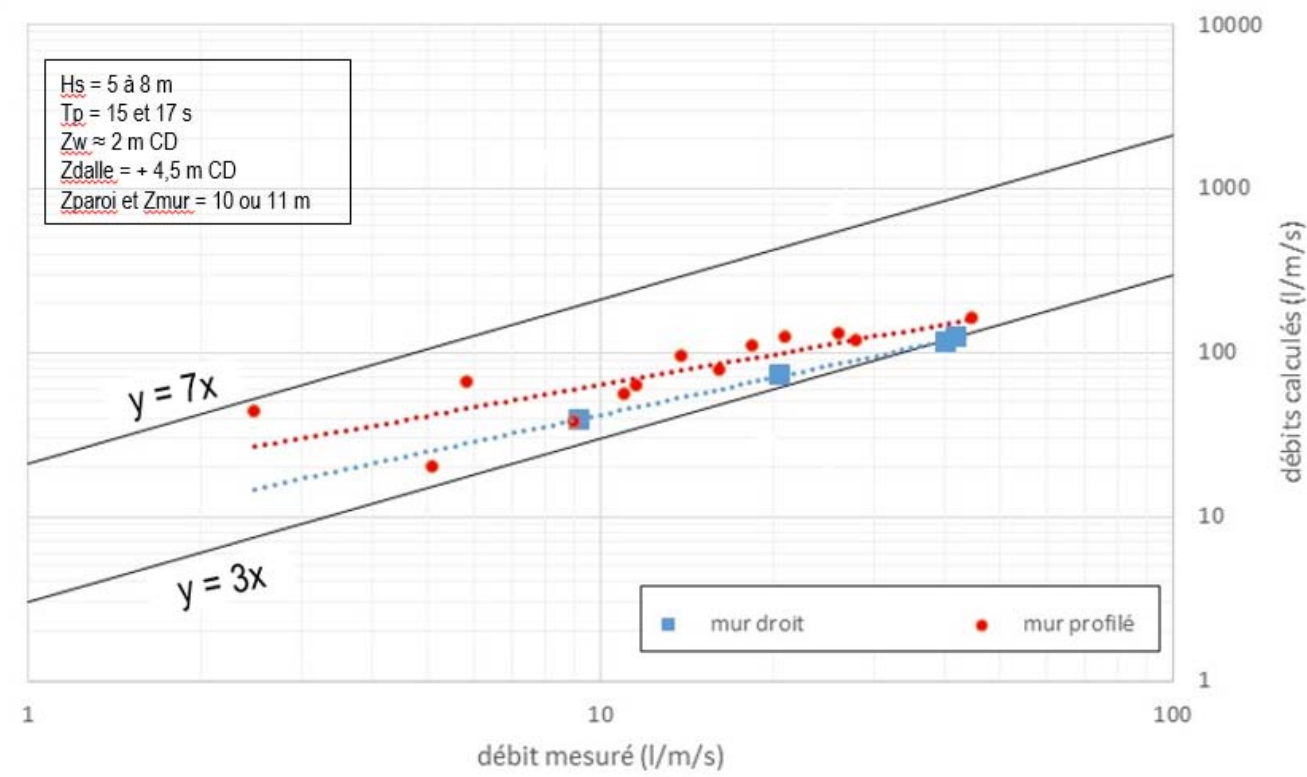

Figure 6. Débits de franchissement associés aux caissons à chambre perchée. 
On constate que, pour les gammes de paramètres testées, la chambre perchée réduit les franchissements d'un facteur compris entre 3 et 7 , et que le profilage du mur arrière a un effet sensible.

Si l'on se réfère à la formule $\mathrm{q}=\mathrm{A} \exp \left(-2.78 \mathrm{R}_{\mathrm{c}} / \mathrm{H}_{\mathrm{s}}\right)$ donnant le débit de franchissement d'un mur vertical attaqué de manière quasistatique (ce qui est le cas vu les fortes périodes $T_{p}$ ), une réduction d'un facteur $\alpha$ correspond à une augmentation de la cote d'arase égale à $\Delta \mathrm{R}_{\mathrm{c}}=\mathrm{H}_{\mathrm{s}} \mathrm{Ln}(\alpha) / 2.78$, soit pour un Hs moyen de $6.5 \mathrm{~m}$ et un facteur moyen $\alpha=5$, une surélévation de $3.5 \mathrm{~m}$.

La chambre perchée permet donc de réduire significativement la cote d'arase des ouvrages de protection, ce qui est particulièrement intéressant dans un contexte urbain.

\subsection{Dispositifs réduisant les vagues en amont}

Dans certains cas où les houles du large attaquent directement le front de mer à aménager, il peut être intéressant de réduire les hauteurs de vagues en amont du projet. La première solution consiste à provoquer le déferlement des plus hautes vagues sur une structure immergée. Comme cet écrêtement suppose une largeur minimale, la technique la plus simple consiste à réaliser un platier artificiel sous-marin, comme dans le cas du front de mer de Beyrouth, dont on voit le modèle physique sur la photo de la figure 7 (laboratoire de Delft Hydraulics). On distingue la ligne de caissons décrite plus haut, protégée en amont par un platier arasé à $-6 \mathrm{~m} \mathrm{ZH}$, et large d'une centaine de mètres (soit environ une demi-longueur d'onde en pied de platier).

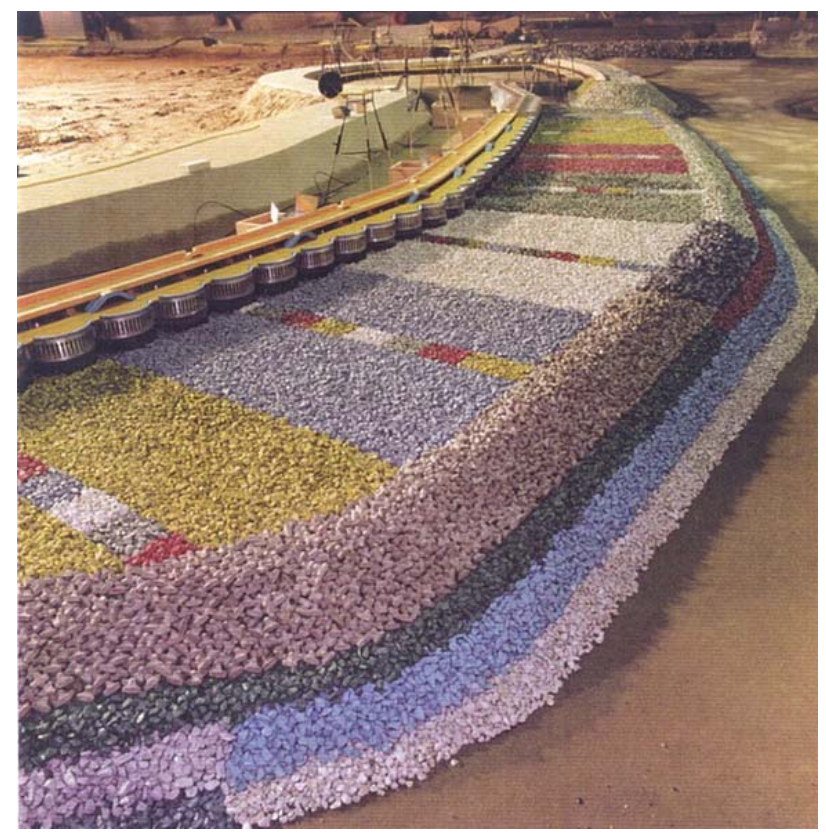

Figure 7. Essai 3D du front de mer de Beyrouth (Delft Hydraulics). 
Cette solution n'est toutefois pas adaptée à des grandes profondeurs, pour lesquelles on doit rechercher des dispositifs plus compacts.

Un concept intéressant, imaginé il y a des années pour Monaco (GUEVEL et al., 1985), et étudié pour le front de mer de Beyrouth à la place du platier précédent puis abandonné lorsque l'on a appris la présence de karsts qui rendait problématique un ancrage par pieux. Il consiste en l'interposition d'une simple plaque rigide placée horizontalement légèrement sous le niveau d'eau au repos.

Ce procédé de "mur d'eau oscillant" est illustré par la modélisation dont les résultats sont montrés sur la figure 8, laquelle a été effectuée avec le code IH2VOF de l’Institut Hydraulique de Cantabrie, qui montre une atténuation significative en aval de la plaque (à droite) moyennant la création d'un mouvement de piston horizontal sous la plaque et une réflexion de la vague en partie supérieure sur le bord aval de la plaque.

Le mur d'eau oscillant permet donc, dans des mers à faible marnage, d'atténuer sensiblement les houles dès lors que leur longueur d'onde est inférieure à environ 5 largeurs de plaque. On obtient un effet intéressant lorsqu'on dispose deux plaques identiques à une distance optimale l'une de l'autre : le coefficient de transmission global est alors inférieur au carré du coefficient de transmission obtenu avec une seule plaque, du fait d'un phénomène de réflexion de Bragg entre les deux barrières (figure 9).

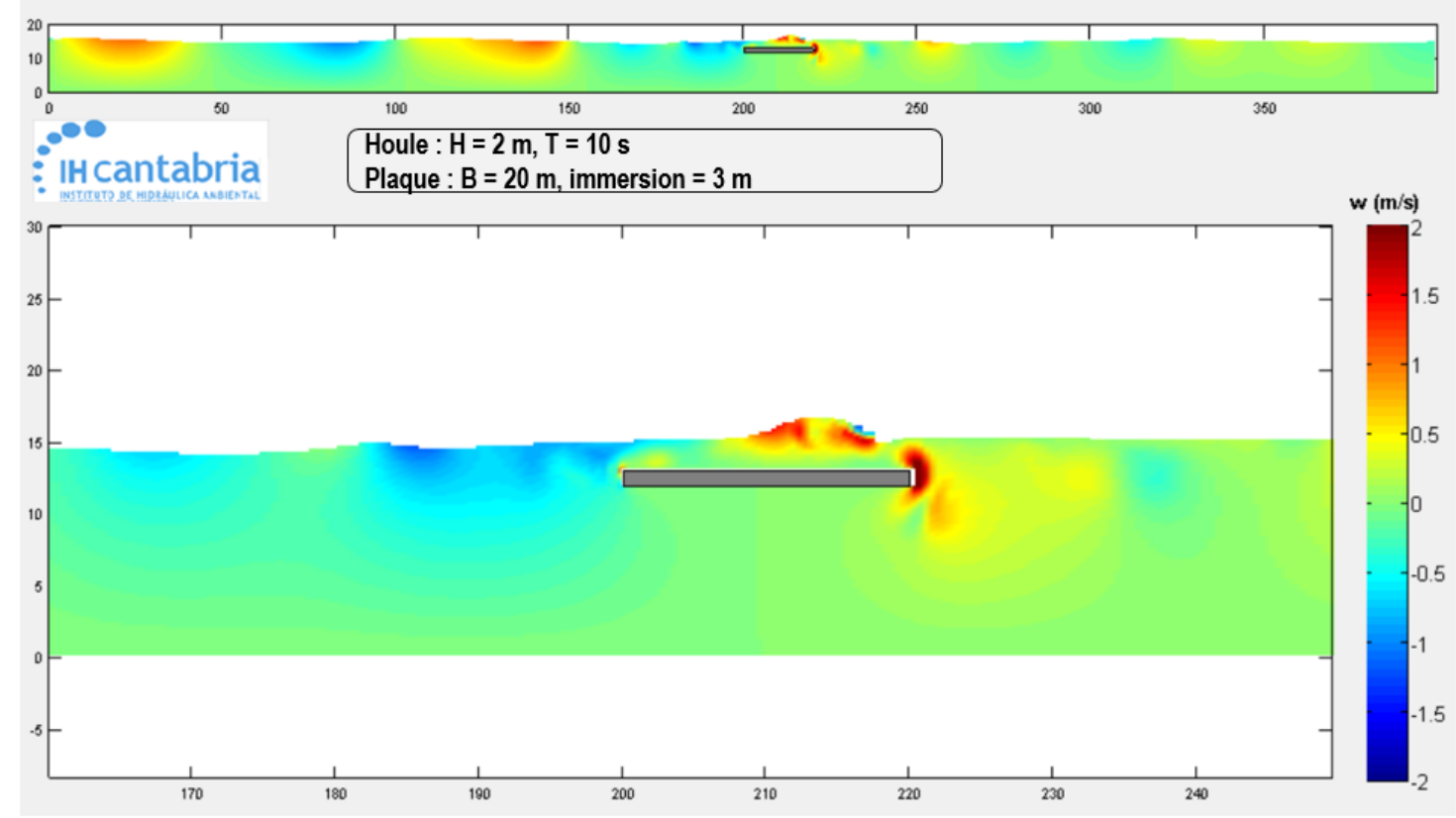

Figure 8. Modèle IH2VOF d'un mur d'eau oscillant. 


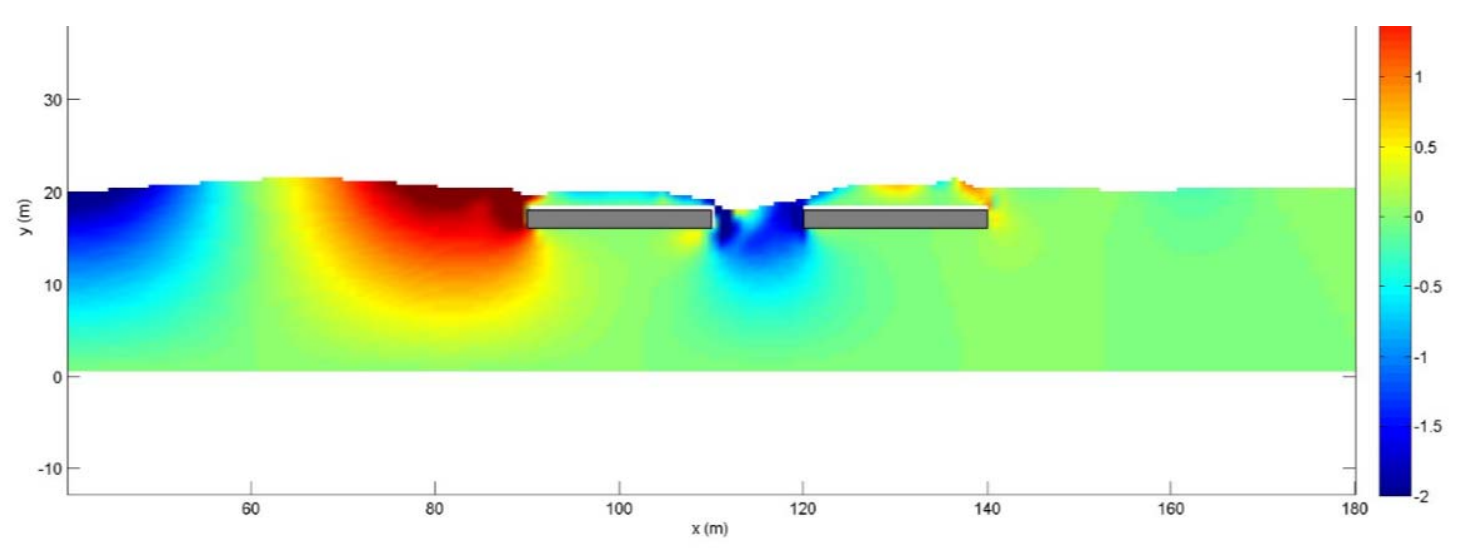

Figure 9. Modèle IH2VOF d'un double mur d'eau oscillant.

\section{Conséquences structurelles des faibles cotes d'arase}

\subsection{Impacts des vagues à l'intérieur des structures}

Les concepteurs de structures offshore font en général en sorte que les ponts de leurs structures soient situés au-dessus de l'élévation maximale des vagues. Dans le cas des extensions urbaines en mer, on a vu qu'il n’était en général pas possible de respecter cette condition pour des raisons architecturales. Cela conduit à la nécessité d'évaluer et de gérer les sous-pressions dynamiques qui s'appliquent aux sous-faces des dallespromenades fermant les chambres de dissipation. Des formules existent pour les sousfaces de jetées sur pieux en mer (voir par exemple ALLSOP et al., 2001), mais pas dans le cas de géométries complexes comme celles décrites plus haut.

Les essais avec mesures de pressions ou d'efforts locaux restent encore la seule méthode fiable pour évaluer les pics de pression de très courte durée. Encore faut-il pouvoir interpréter les mesures brutes de pression $\mathrm{P}_{\mathrm{i}}(\mathrm{t})$, et notamment savoir répondre aux deux questions suivantes :

- sur quelle surface les intégrer à un instant t donné ?

- les pressions mesurées suivent-elles la similitude de Froude?

La seconde question se ramène à la distinction entre :

- impact ventilé, auquel cas la similitude de Froude s'applique, et où la surface d'application dépend de la géométrie du caisson, de la cinématique de l'écoulement et de la durée du pic de pression mesuré; pour apprécier la surface d'application instantanée, on peut s'appuyer sur les pressions concomitantes mesurées par un capteur voisin, ou sur une mesure d'effort locale, qui par nature intègre les pressions sur une surface représentative (en n’oubliant pas la correction éventuelle de fréquence propre et d'amortissement du capteur).

- impact avec piégeage d'une poche d'air entre la vague et les parois, auquel cas la pression réelle est significativement réduite par rapport à la similitude de Froude, du fait de la prise en compte de la compressibilité de l'air à la pression atmosphérique 
(LUNDGREN,1969). La pression ainsi obtenue s’applique alors simultanément sur toute la surface susceptible de fermer la poche d'air.

Pour distinguer en pratique, outre le fait qu'un "compression shock" est en général suivi d'oscillations rapides de la pression, il est judicieux de disposer de plusieurs capteurs de pression et d'observer si le déphasage entre les pics mesurés est cohérent avec une célérité proche de celle des ondes de gravité (de l'ordre de $1 \mathrm{~m} / \mathrm{s}$ en modèle réduit) ou de celle des ondes sonores dans le liquide (de l'ordre de 100 à 1500 m/s selon le degré d'aération).

\subsection{Impacts des vagues sur la face supérieure des structures}

La nécessité de construire des structures de faible hauteur au-dessus du niveau d'eau conduit également à des problématiques qui s’apparentent au "green water impact" sur les ponts de bateaux.

Ceci est illustré par le caisson-piscine qui a été imaginé pour l'Anse du Portier à Monaco (ARISTAGHES et al., 2018), dont la fonction première était la protection du port intégré dans le projet, et qui est devenu un caisson très bas au-dessus de l'eau (crête à + 2.95 m NGF pour des niveaux d'eau allant de -0.3 à + 1.8 NGF).

La figure 10 montre le caisson (les "fentes" côté large sont en fait des vitres en plexiglas, donc constituent une paroi verticale fermée), la maquette en position piscine couverte.
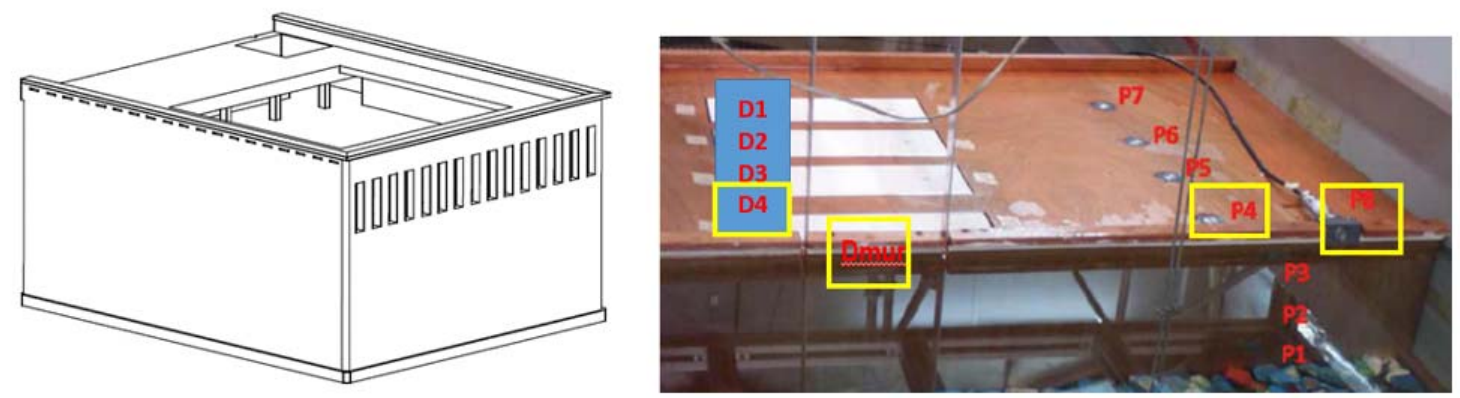

Figure 10. Caisson piscine et instrumentation (Oceanide)

Des pics de pression très violents sur la dalle (capteur de pression P4 et d'effort sur une dalle instrumentée) ont été mesurés pour plusieurs vagues retombant d’une hauteur d'environ 10 m au-dessus de la dalle, comme représenté sur la figure 11. 

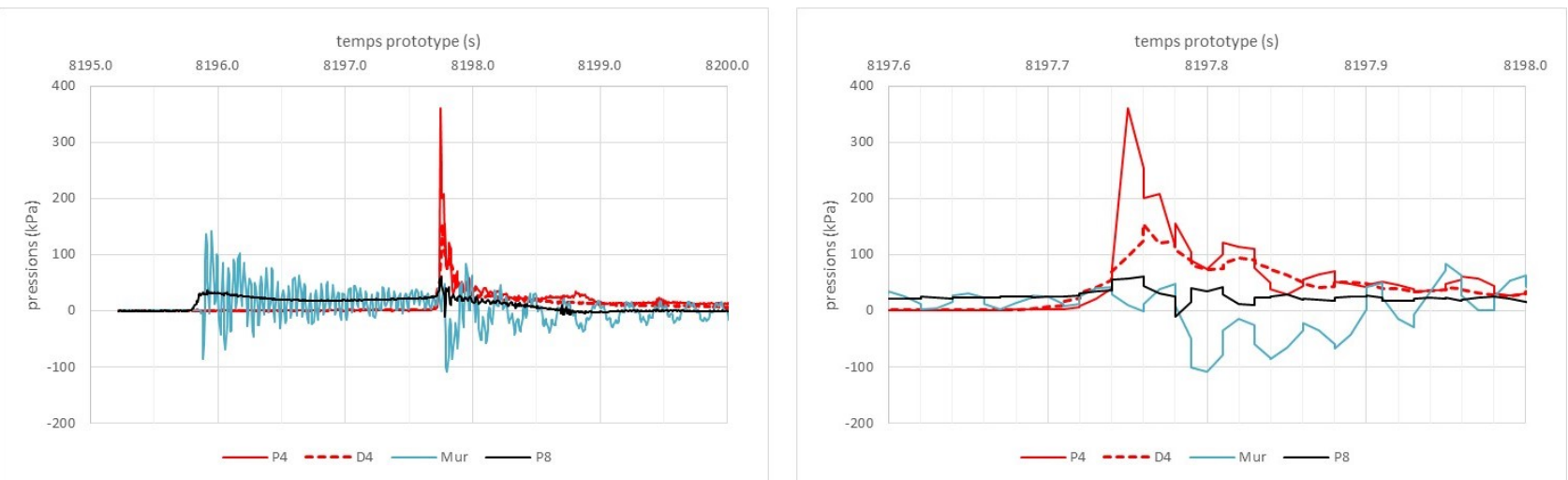

Figure 11. Pressions mesurées lors d'une retombée de franchissement.

On observe d'abord ( $\mathrm{t}=8196 \mathrm{~s})$ un palier à $30 \mathrm{kPa}$ sur le capteur de pression P8 placé sur le mur chasse-mer, associé à une pression moyenne similaire sur le capteur d'effort $\mathrm{D}_{\text {mur }}$ (avec des oscillations liées à la période propre de la balance supportant la partie de mur instrumentée).

On note ensuite un pic brutal $1.8 \mathrm{~s}$ plus tard sur les capteurs placés sur la dalle (P4 et D4), suivi de pics de moins en moins marqués et décalés dans le temps de manière régulière sur les autres capteurs positionnés à distance croissante du front.

Lorsque l'on analyse plus en détail ce pic (graphique de droite), on note que le capteur d'effort D4 mesure une pression moyenne (sur $3 \mathrm{~m}$ de largeur) pratiquement divisée par 2 par rapport au pic de pression P4. On observe également un pic sur le capteur d'effort $\mathrm{D}_{\text {mur}}$, orienté cette fois vers le large, et dont la valeur réelle nécessite de retraiter le signal en tenant compte des périodes propres et amortissements du capteur.

Des essais similaires ont été conduits avec la piscine ouverte, et ont conduit à des pressions sous environ $2 \mathrm{~m}$ de matelas d'eau d'ordre de grandeur similaire à celles mesurées au même endroit. Plus précisément, le pic mesuré en $\mathrm{P} 5$, situé $6 \mathrm{~m}$ derrière le capteur P4, passe selon les houles testées de 60-120 kPa dans le cas d'une dalle béton à 50-80 kPa en fond de piscine).

On peut approcher les ordres de grandeur de ces pressions en fond de piscine sous $2 \mathrm{~m}$ de matelas d'eau, en étendant le modèle de Wagner d'impact d'un corps en chute libre sur un plan d'eau (MOLIN, 2002), en considérant que le fond de la piscine est un plan de symétrie, et en cumulant la chute d'une lame d'eau d'épaisseur métrique tombant d'une hauteur de $10 \mathrm{~m}$ avec la "chute vers le haut" d'une lame identique sur un plan d'eau virtuel situé $2 \mathrm{~m}$ sous le fond de la piscine.

\subsection{Gestion des franchissements}

Le faible niveau d'arase conduit à des débits de franchissements significatifs sous événement extrême (typiquement de durée de retour 50 ou 100 ans). 
Cela conduit, à la nécessité d'imaginer des systèmes de drainage à la fois efficaces et architecturalement acceptables.

On peut également envisager le recours à des matériaux d'un type nouveau, comme les bétons à très forte perméabilité.

Cela amène également à tenter de mieux définir les conditions limites d'exploitation, en se donnant des couples houle - niveau d'eau conduisant conjointement à une durée de retour donnée $\mathrm{R}$, plutôt que de combiner houle et niveau de durée de retour $\mathrm{R}$, comme souvent pratiqué pour des dimensionnements structurels d'ouvrages.

Raisonner autrement conduirait à diminuer la relation de la zone urbaine à la mer, ce qui n’est pas le but recherché, et ceci sans que ce soit réellement nécessaire.

La méthode du British Department of Environment, Food and Rural Affairs (HAWKES, 2005) a le mérite d'être simple. Elle consiste à caractériser le degré de corrélation entre hauteur $\mathrm{H}_{\mathrm{s}}$ et niveau d'eau $\mathrm{Z}_{\mathrm{w}}$ maximaux sur un cycle de $12 \mathrm{~h}$ par un coefficient $\mathrm{C}$ défini par :

$$
C=\frac{p\left(H_{s}>h \text { et } Z_{w}>z\right)}{p\left(H_{s}>h\right) \cdot p\left(Z_{w}>z\right)}
$$

Ce coefficient peut être évalué si l'on dispose de données concomitantes de houles et de niveaux sur une période suffisamment longue. On en déduit alors des diagrammes similaires à la figure 12 , donnant les couples de durées de retour $\mathrm{R}_{\mathrm{Hs}}$ et $\mathrm{R}_{\mathrm{Zw}}$ associés à une durée de retour globale donnée.

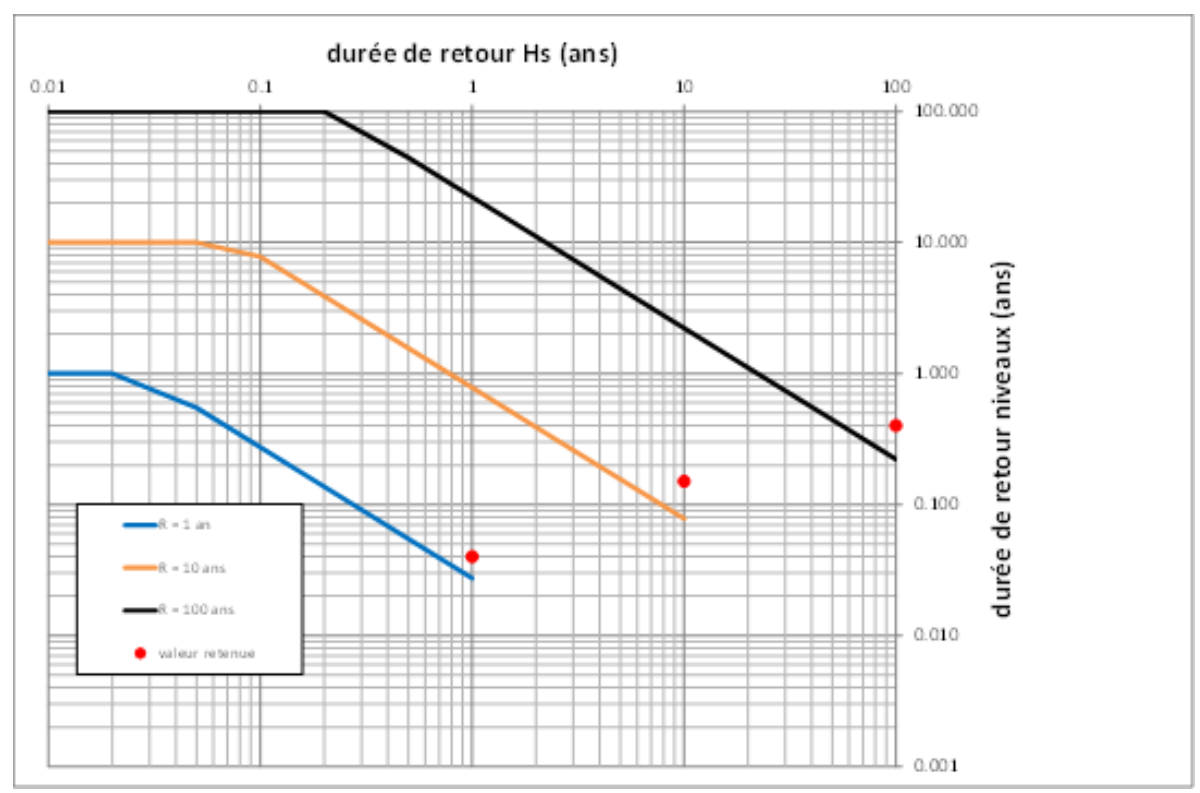

Figure 12. Durées de retour houles et niveaux concomitants. 


\section{Conclusion}

Les extensions urbaines en mer sont appelées à se généraliser. Or elles demandent des solutions et approches nouvelles. On peut raisonnablement penser que dans les prochaines années des recherches et innovations porteront sur des dispositifs antifranchissements plus compacts ou moins visibles, et que les méthodes de prédiction des franchissements et des efforts induits s'étendront à ces nouveaux concepts.

\section{Références}

ALLSOP W. et al. (2001). Guidelines for the hydraulic design of exposed jetties. HR Wallingford.

ARISTAGHES P, BLANCHET V., COMPTE D., GREVOZ A., QUENOUILLE B., TELES M., WEULERSSE A. (2018). L'extension en mer de l'Anse du Portier à Monaco. XV ${ }^{\text {èmes }}$ Journées Nationales Génie Côtier - Génie Civil, La Rochelle, pp 409420. https://doi.org/10.5150/jngcgc.2018.046

ARISTAGHES P., CHALOIS T. (2007). Beirut Sea Front Hydraulic Design. Ports2000, Barcelone.

BOUCHET R. (2007). Digue de Monaco et urbanisation en mer. JST CETMEF, Paris. GUEVEL P., LANDEL E., BOUCHET R., MANZONE J.M. (1985). Le phénomène d'un mur d'eau oscillant et son application pour protéger un site côtier. ATMA.

HAWKES P.J. (2005). Use of Joint Probability Methods in Flood Management. UK Defra / Environment Agency.

LUNDGREN H. (1969). Wave shock forces. Techn Uniersity of Denmark.

MOLIN B. (2002). Hydrodynamique des Structures Offshore. Section 7.2, EditionsTechnip.

EUROTOP (2016). Manual on wave overtopping of sea defences and related structures. 